\title{
A putative HIV-1 subtype C/CRF11_cpx unique recombinant from South Africa
}

\author{
Pascal Obong Bessong ${ }^{1 *}$ and Benson Iweriebor ${ }^{2}$
}

\begin{abstract}
The HIV epidemic in South Africa is overwhelmingly driven by HIV-1 subtype C viruses. The HIV gag, pol, env (C2-V5) and nef sequences of virus 08MB26ZA, obtained from a 47 year old woman, were studied by phylogenetic analysis, REGA and the jumping Profile Hidden Markov Model (jPHMM) tools. The pol gene was further analyzed for recombination by Simplot. The pol and env sequences were examined for genetic drug resistance mutations and predicted co-receptor usage respectively. There was agreement in the assignment of the gag sequence as pure HIV-1 subtype $\mathrm{C}$ by phylogenetic, REGA and jPHMM analyses. The pol sequence clustered with CRF11_cpx and in the J-clade by phylogenetic analysis; and to a CRF11_cpx/subtype C recombinant by REGA. The assignment of po/ to CRF11_cpx and subtype $C$ was confirmed by Simplot. The recombinant was of the R5 biotype, with no important drug resistance mutations in the pol region. The epidemiologic and biologic significance of the virus are unknown. The finding suggests that complex viruses are being introduced into South Africa with potential implications for diagnosis. This is apparently the first report from South Africa of a putative unique recombinant involving CRF11_cpx and subtype C genomes.
\end{abstract}

Keywords: HIV-1 subtype C, CRF11_cpx, Recombinant, HIV subtyping, South Africa

\section{Background}

Genetic variants of HIV contribute to the pandemic worldwide and have potential consequences for diagnostics, treatment and treatment monitoring, and the development of an effective vaccine (Hemelaar 2013). Several factors account for the high genetic diversity of HIV: rapid replication turnover, in vivo host selective immune and treatment pressure, and recombination events during replication (Liitsola et al. 1998; Corbet et al. 2000; Ramirez et al. 2008; Taylor et al. 2008). Due to this variability, HIV is classified into types 1 and 2; with type 1 further classified into groups $\mathrm{M}, \mathrm{O}, \mathrm{N}$ and $\mathrm{P}$. Group $\mathrm{M}$, which is responsible for the global HIV-1 epidemic, comprises nine recognized phylogenetic subtypes (A-D, $\mathrm{F}-\mathrm{H}, \mathrm{J}$, and $\mathrm{K}$ ), with further divisions into sub-subtypes for $\mathrm{A}$ and $\mathrm{F}$ viruses.

\footnotetext{
*Correspondence: pascal.bessong@univen.ac.za; pascal.bessong@gmail. com

${ }^{1}$ HIV/AIDS and Global Health Research Programme, Room FF172 Life Sciences Building, Department of Microbiology, University of Venda, Thohoyandou 0950, South Africa

Full list of author information is available at the end of the article
}

The emergence of circulating recombinant forms (CRFs) in the global HIV-1 pandemic is important, as together with unique recombinant forms (URF) represent about $20 \%$ of infections (Hemelaar et al. 2011). A complex (cpx) variant represents a recombinant derived from genes of three pure subtypes. CRF11_cpx involving subtypes A, G, J and CRF01-AE is observed in the Republic of Congo, Cameroon and Central African Republic (Wilbe et al. 2000; Montavon et al. 2002). Analysis of partial sequences suggests the presence of CRF11_cpx in Senegal and Gabon (Paraskevis et al. 2000). Subtype J was discovered in 1995 and originated most probably in the Democratic Republic of Congo. Few subtype J sequences are available, and these are mostly from the Democratic Republic of Congo, Cameroon, and Senegal (Los Alamos Database 2014). However, fragments of subtype J are present in many mosaic recombinant forms originating from West Africa (CRF06_cpx) and Central West Africa (CRF11_cpx, CRF13_cpx, and CRF49_cpx), suggesting that this subtype, either in a pure or in a recombinant form, is probably prevalent across Central and West Africa (Laukkanen et al. 1999). Subtype J sequences have

\section{Springer}

(c) 2016 Bessong and Iweriebor. This article is distributed under the terms of the Creative Commons Attribution 4.0 International License (http://creativecommons.org/licenses/by/4.0/), which permits unrestricted use, distribution, and reproduction in any medium, provided you give appropriate credit to the original author(s) and the source, provide a link to the Creative Commons license, and indicate if changes were made. 
also been reported in Uganda, Angola, Zambia, Cuba, Spain and France (Trask et al. 2002; Yebra et al. 2013; Bartolo et al. 2009; Kiwanuka et al. 2009).

Human immunodeficiency type 1 subtype $C$ is overwhelmingly responsible for the epidemic in South Africa, although over time, other subtypes such as A, D, CRF01_ $\mathrm{AE}$, inter-subtype and URF such as $\mathrm{A} 1 / \mathrm{C}, \mathrm{A} 1 / \mathrm{D}$ have been identified (Bredell et al. 2002; Iweriebor et al. 2011; Wilkinson and Engelbrecht 2009). However, CRF11_cpx has not been reported from South Africa. In this report, a plausible recombinant, comprising sequences from HIV-1 subtype C and CRF11_cpx identified in a South African patient is described.

\section{Results and discussion}

\section{Phylogenetic analyses of gene regions}

In an initial phylogenetic analysis, the partial pol sequence [complete protease (PR) and first $900 \mathrm{bp}$ of the reverse transcriptase (RT)] clustered with CRF11_cpx reference sequences. Additional recombination analysis with REGA tool revealed a mosaic pattern of unclassified regions alternating with subtype J regions (Nwobegahay et al. 2011). Consequently, an attempt was made to further elucidate the genetic makeup of the virus.

Attempted amplification of the complete genome of sample 08MB26ZA was not successful. Hence gene fragments were generated and directly sequenced and the following reliable sequence lengths were obtained: gag sequence (1436 nucleotides, position 789-2252), pol (2506 nucleotides, position 2252-4758), env C2-V5 (719 nucleotides, position 7100-7819), and nef (616 nucleotides, position 8792-9413), positions with reference to HXB2 nucleotide coordinates. Phylogenetically, the gag sequence clustered with HIV-1 subtype C reference sequences with a bootstrap value of $70 \%$ (Fig. 1).

The pol gene (i.e. PR + RT + integrase (IN)) clustered with CRF11_cpx sequences with a bootstrap value of $98 \%$, in the subtype J clade (Fig. 2a). However, without the IN region, the pol (i.e. PR + RT) clustered with CRF11_cpx sequences (Fig. 2b). In addition, the PR, RT and IN sequences were individually analyzed: the PR and RT sequences clustered with CRF11_cpx (Figs. 3, 4); and the IN sequence is related to HIV- 1 subtype $C$ sequences, but this was not supported by a high bootstrap value (Fig. 5). The env (C2-V5) sequence showed a close relation to subtype $\mathrm{C}$ sequences with a bootstrap value of $77 \%$, but not definitively typable (Fig. 6), while the nef sequence clustered with subtype $\mathrm{C}$ reference sequences (Fig. 7).

\section{Subtype and recombination analyses of gag and pol genes by REGA}

Upon HIV subtype analysis with the REGA tool, the gag sequence was assigned to HIV-1 subtype $\mathrm{C}$ with a bootstrap confidence of $>70 \%$ (Fig. 8). On the other hand, the pol sequence was shown to contain sequences from subtype J, G, and C in an alternating fashion, with a bootstrap confidence of $>70 \%$ (Fig. 9a). Subsequent HIV subtype and CRF recombination analysis with REGA showed that the pol was related to both CRF11_cpx (segment 1, Fig. 9b); and HIV-1 subtype C (segment 2, Fig. 9b). The CRF11_cpx relatedness of the pol sequence is represented by the PR and RT regions, and the HIV-1 subtype $\mathrm{C}$ relatedness is represented by the IN region. This is in agreement with the outcomes of the maximum likelihood phylogenetic analyses of the PR, RT, combined PR and RT, and the IN regions.

The assignment of the pol sequence as related to CRF11-cpx/C was according to the REGA schedule 3A which is based on 4 parameters: the query sequence greater than $800 \mathrm{bp}$, clustering with a CRF with a bootstrap confidence greater than $70 \%$, coupled with the detection of recombination in the pure subtype bootscan, and further confirmed by CRF bootscan analysis with a confidence of more than $90 \%$ (Peña et al. 2012).

\section{Subtype and recombination analysis by jPHMM and Simplot}

The gene fragments were also subjected to jumping Profile Hidden Markov Model (jPHMM) and Simplot analyses. On jPHMM analysis, the gag, env and nef sequences were assigned as related to subtype $\mathrm{C}, \mathrm{PR}$ as related to subtype J, RT as J/C; and IN as C/A1. The pol $(\mathrm{PR}+\mathrm{RT}+\mathrm{IN})$ sequence was determined as recombinant comprising sequences from subtypes $\mathrm{J}, \mathrm{C}$ and $\mathrm{K}$ (Fig. 10). Further bootscan analysis by Simplot showed that the pol contained sequences from CRF11_cpx and subtype $C$ viruses (Fig. 11); in agreement with the outcomes of the REGA, and the phylogenetic analysis in which the pol clustered with CRF11_cpx sequences. A summary of the outcomes of the subtyping and recombination analyses of the different gene regions of 08MB26ZA is shown in Table 1.

There was agreement among the analytic tools in inferring subtypes and recombination patterns for some of the genes regions and not for others. For example, jPHMM indicated the presence of subtype A1 sequences in the IN gene in addition to subtype $C$ sequences; and also the presence subtype $\mathrm{K}$ sequences in the pol gene. The jPHMM indicated the presence of subtype $J$ sequences in the pol gene in contrast to CRF11_cpx indicated by phylogenetic analysis and REGA. This discrepancy can however be reconciled in that subtype J sequences constitute a significant component of CRF11_cpx viruses. Consequently, the indication by phylogenetic analysis, REGA and Simplot tools of the presence of CRF11_cpx sequences in the pol gene, in addition to the assignment 


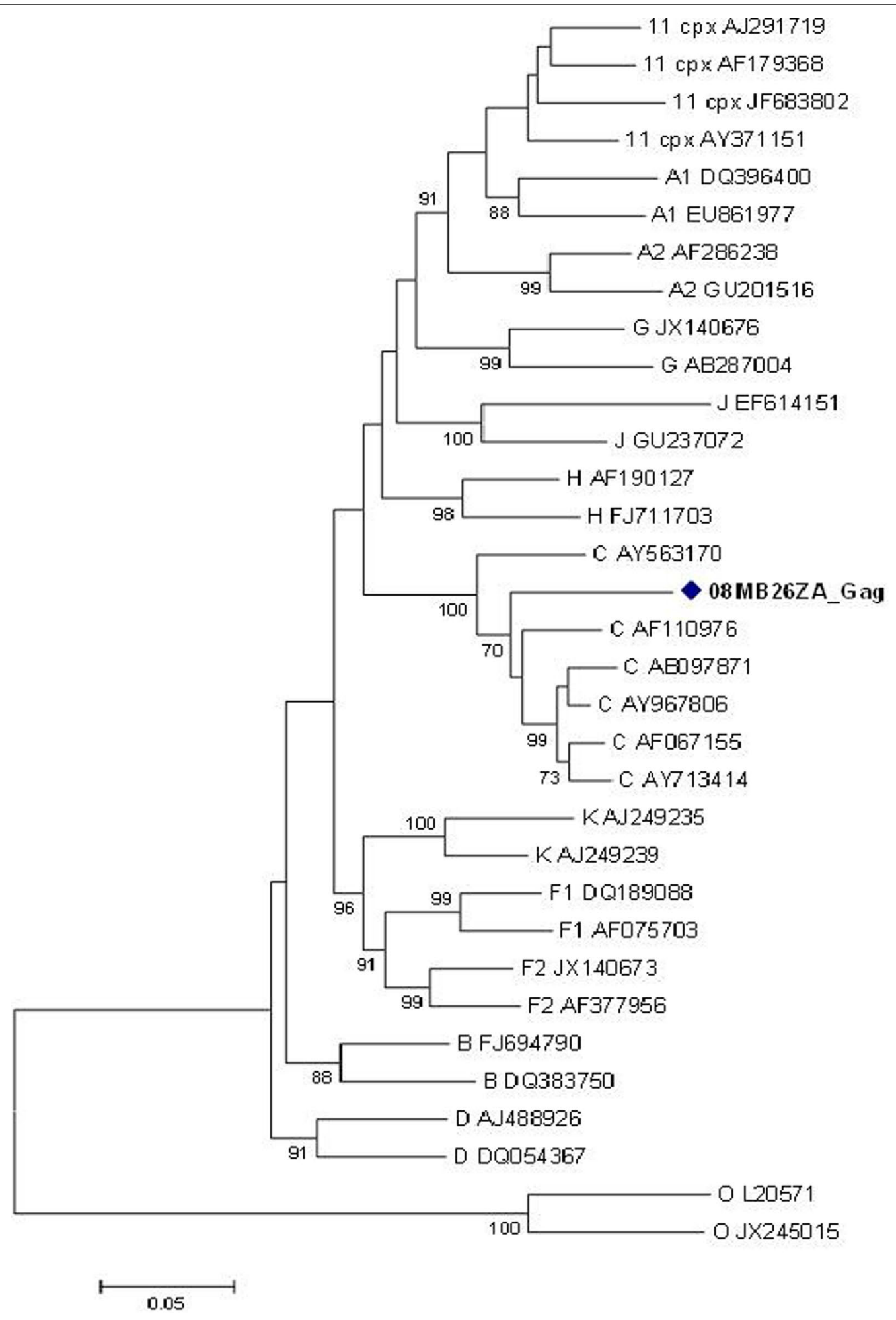

Fig. 1 Maximum likelihood phylogenetic analysis of the gag sequence of virus 08MB26ZA. The sequence is shown to cluster with HIV-1 subtype C reference sequences. Bootstrap values above $70 \%$ are shown. The tree is rooted with group O sequences. The scale represents the number of base substitutions per site 


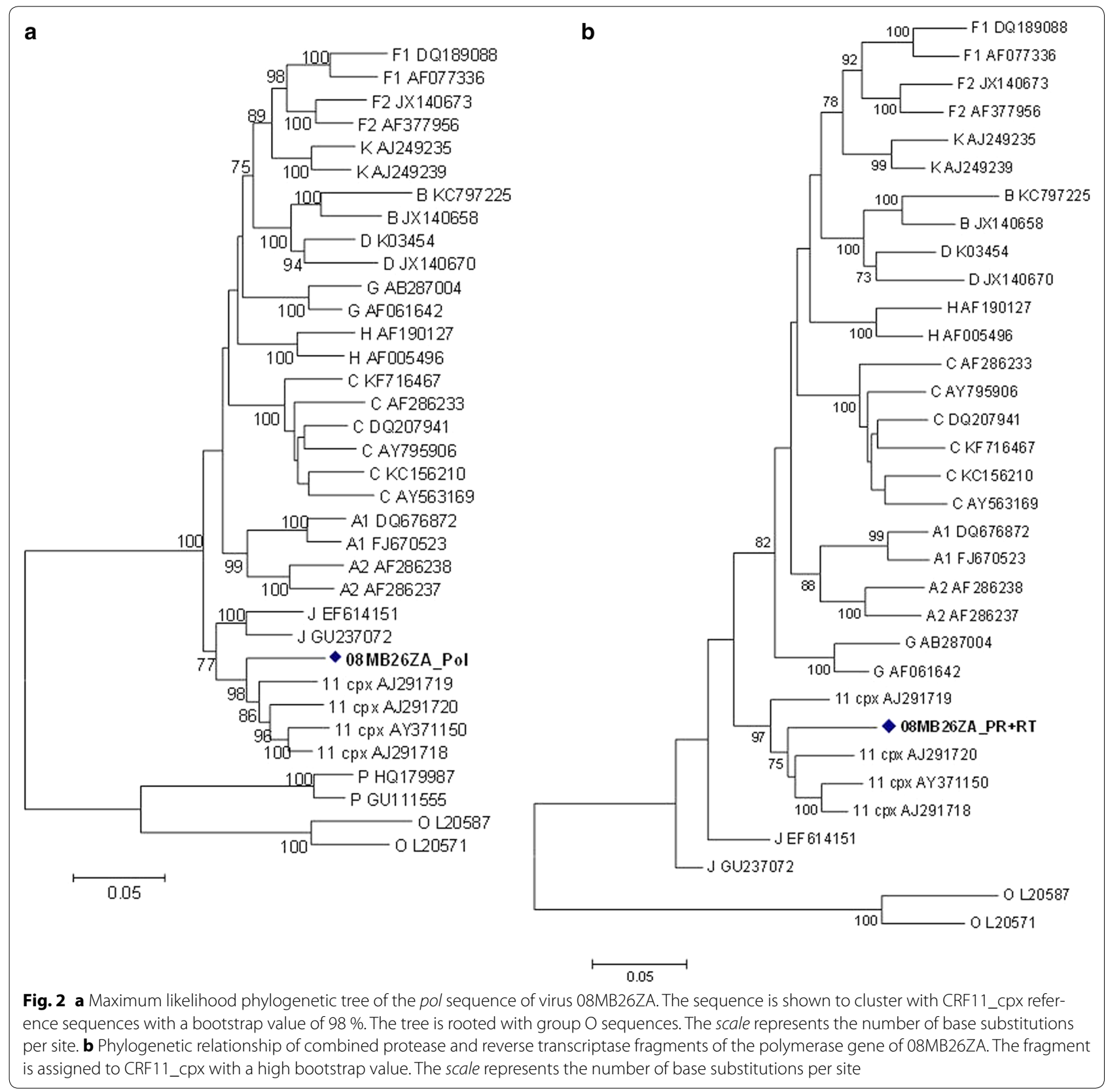

of the gag, env and nef genes to subtype C overwhelmingly suggests that virus 08MB26ZA harboured HIV-1 subtype $C$ and HIV-1 CRF11_cpx sequences.

The partial characterization of a CRF11_cpx/HIV-1 subtype C-like virus from a 47 year old South African female is reported. The current analysis shows that the virus differed markedly from pure HIV-1 subtype J and CRF11_cpx in its sub-genomic assignments. It is only in the PR and RT genes that it exhibited a related pattern of mosaicism as seen in CRF11_cpx. The entire genome of CRF11_cpx is partitioned into sub-genomic regions comprising subtypes A, J, U, G and CRF01_AE (Paraskevis et al. 2000), and in 08MB26ZA subtype $G$ and J sequences were also identified in the pol gene by REGA analysis. The $g a g$ of $08 \mathrm{MB} 26 \mathrm{ZA}$ is related to subtype C, whereas in CRF11_cpx, it is derived from subtype A. Similarly, the IN, env C2-V5 and nef genes of 08MB26ZA are related to subtype $C$, whereas in CRF11_cpx the IN and $e n \nu \mathrm{C} 2-\mathrm{V} 5$ genes are derived from subtype $\mathrm{A}$ and $n e f$ is derived from CRF01_AE. HIV-1 subtype J and CRF11_ cpx are predominantly found in the Central African region (Koyalta et al. 2009; Torimiro et al. 2009; Ndembi 


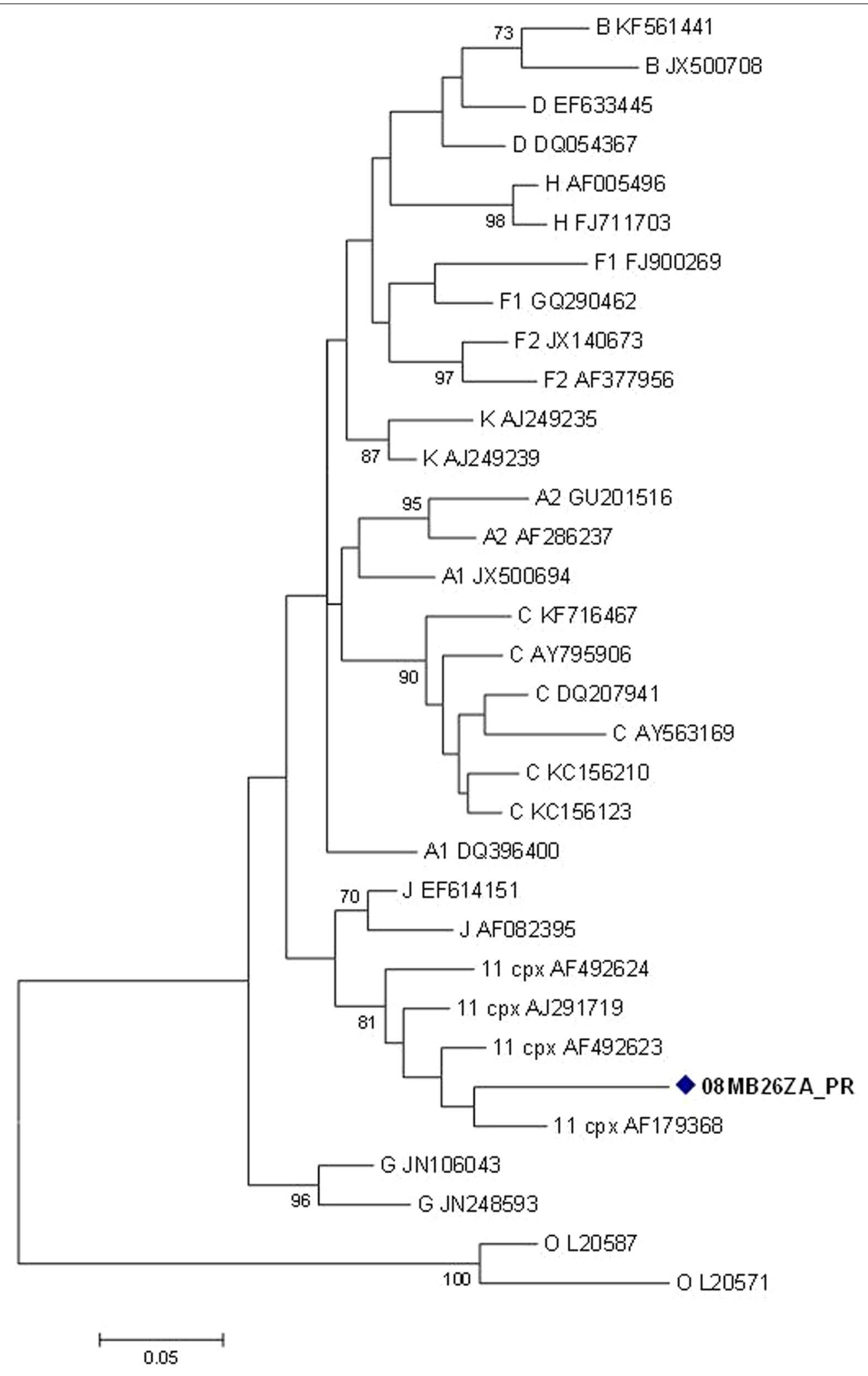

Fig. 3 Maximum likelihood phylogenetic analysis of the protease sequence of virus 08MB26ZA. The sequence is shown to cluster with CRF11_cpx reference sequences. Bootstrap values above $70 \%$ are shown. The tree is rooted with group $\mathrm{O}$ sequences. The scale represents the number of base substitutions per site 


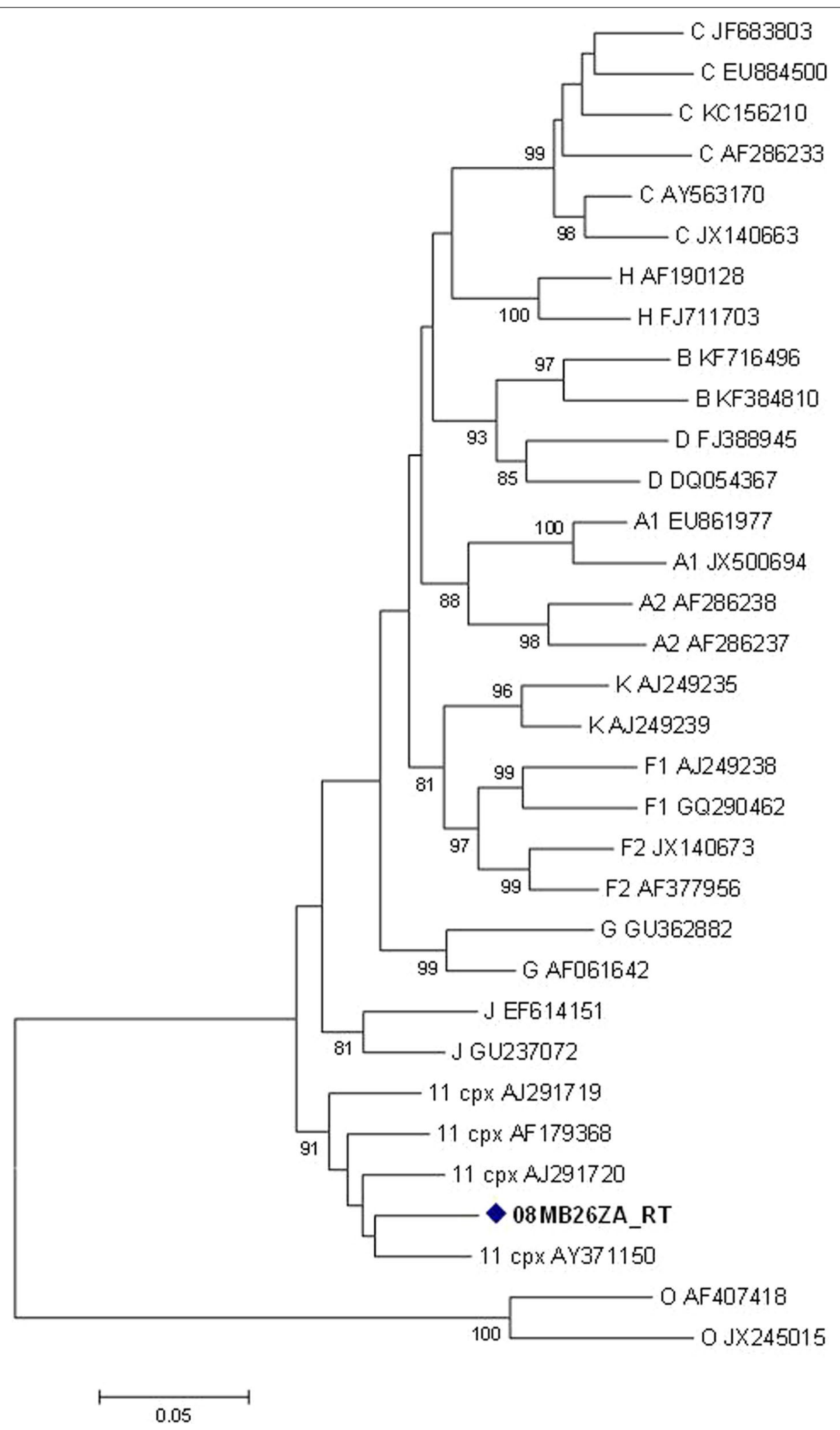

Fig. 4 Maximum likelihood phylogenetic analysis of the RT sequence of 08MB26ZA. The sequence clusters with HIV-1 CRF11_cpx reference sequences. Bootstrap values above $70 \%$ are shown. The tree is rooted with group $\mathrm{O}$ sequences. The scale represents the number of base substitutions per site 


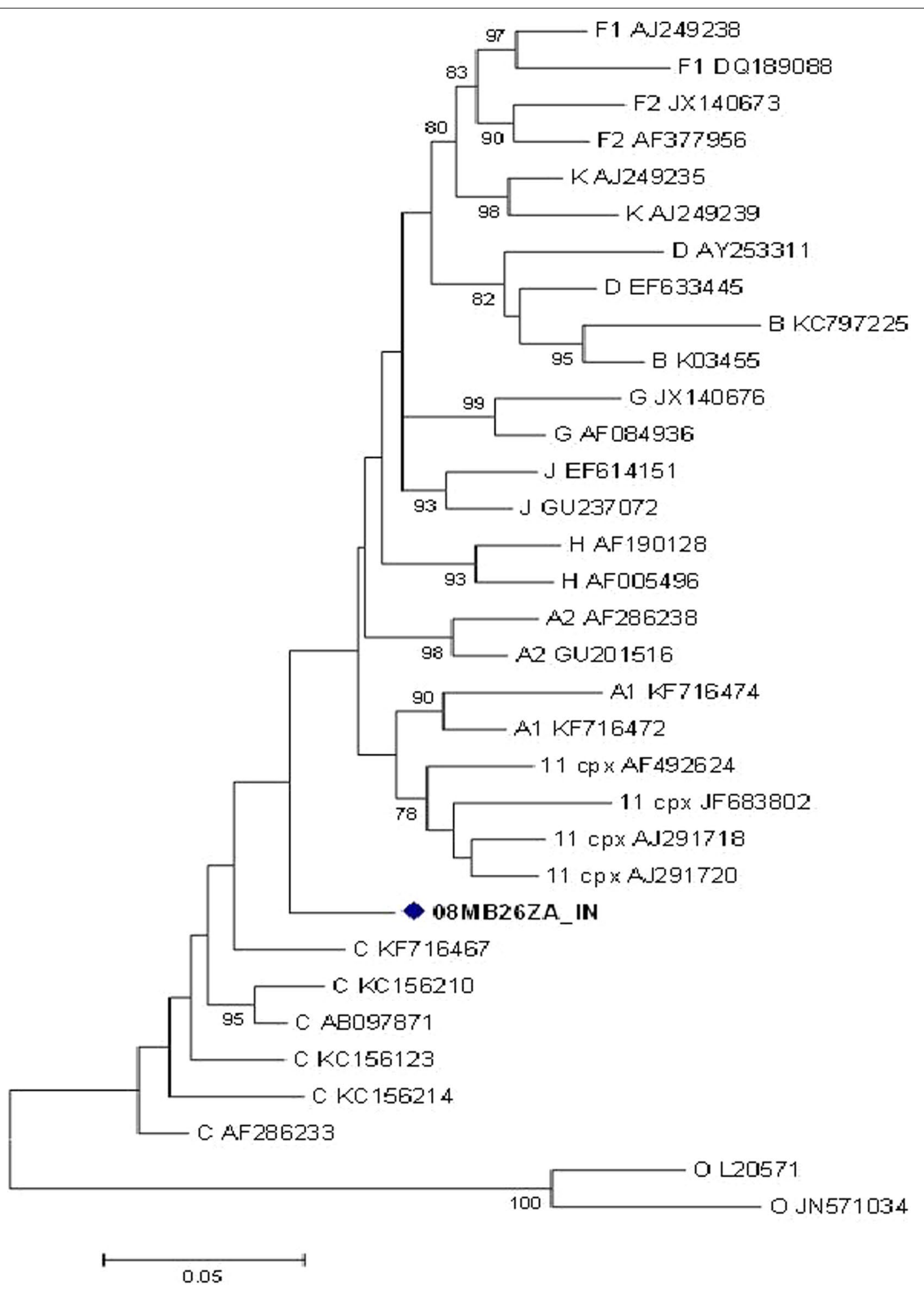

Fig. 5 Maximum likelihood phylogenetic analysis of the integrase sequence of 08MB26ZA. The sequence is not delineated as either related to HIV-1 subtype C or CRF11_cpx reference sequences. Bootstrap values above $70 \%$ are shown. The tree is rooted with group O sequences. The scale represents the number of base substitutions per site 


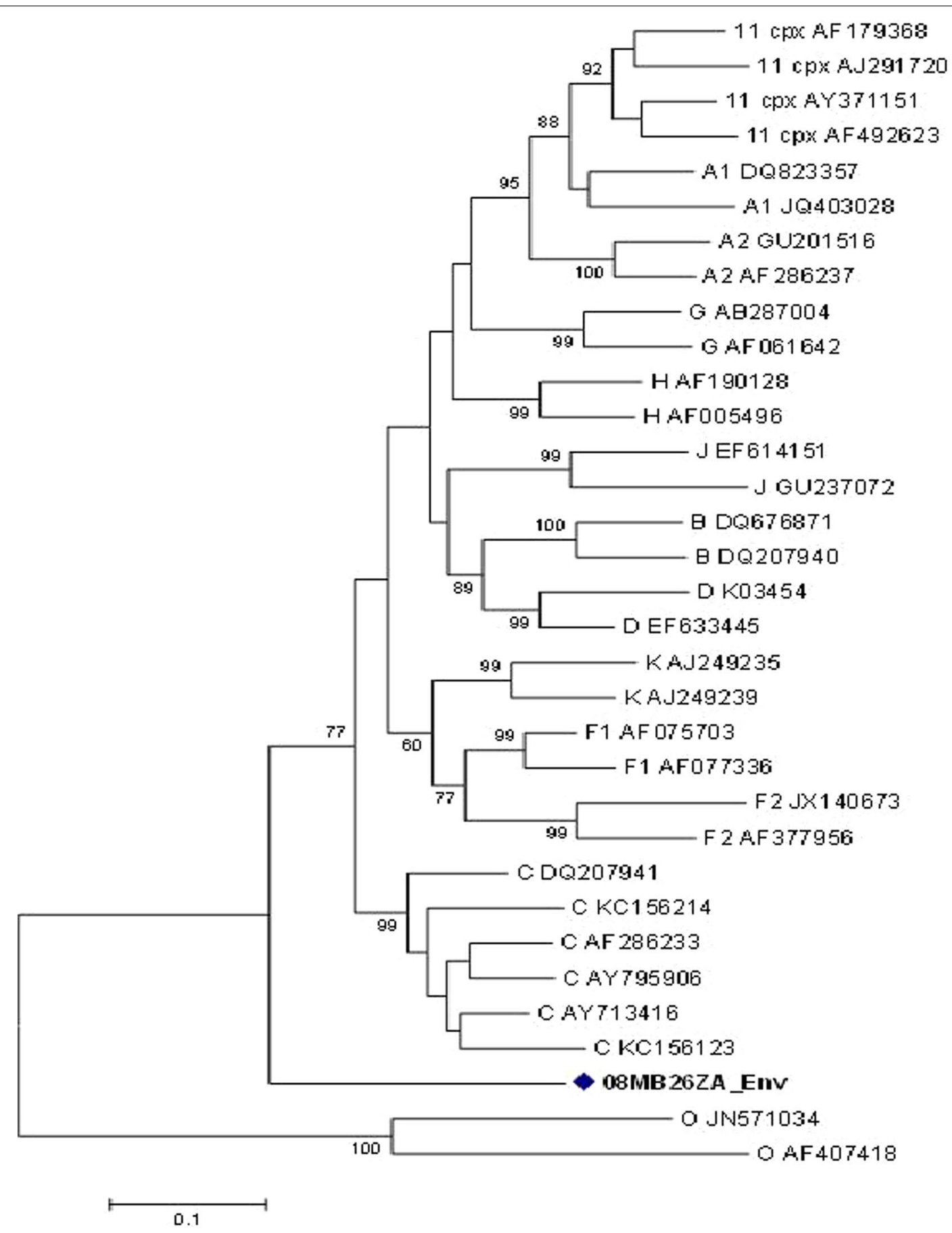

Fig. 6 Maximum likelihood phylogenetic analysis of the env (C2-V5) sequence of 08MB26ZA. The sequence is shown as untypable. Bootstrap values above $70 \%$ are shown. The tree is rooted with HIV-1 group O sequences. The scale represents the number of base substitutions per site

et al. 2008; Caron et al. 2012), with few reports from Southern Africa (Angola and Zambia) on the presence of subtype J viruses (Trask et al. 2002; Yebra et al. 2013; Bartolo et al. 2009).
Availability of the complete genome would have afforded a detailed understanding of the mosaic nature of virus 08MB26ZA allowing for a full comparison with CRF sequences in the sequence database. However, a 


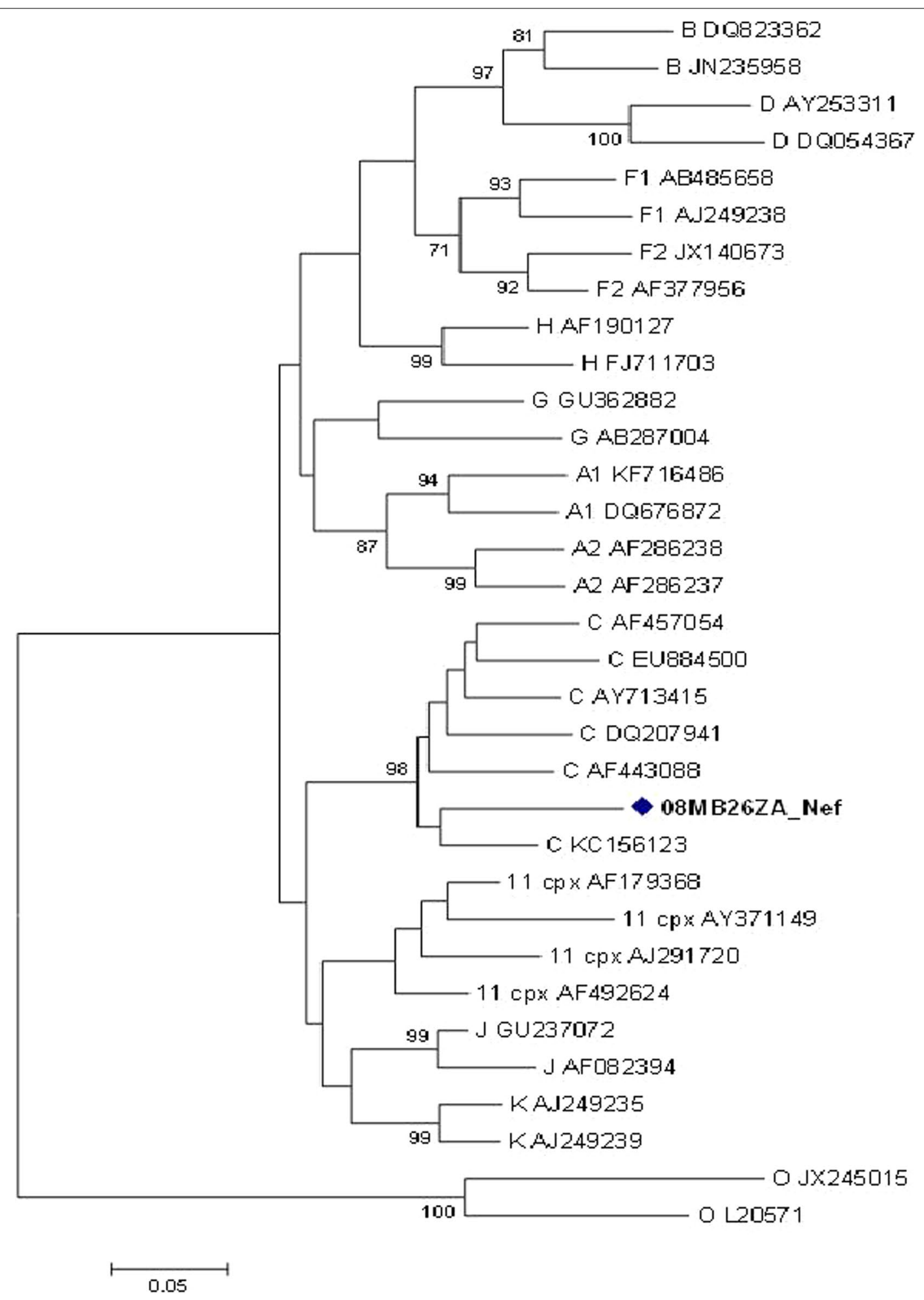

Fig. 7 Maximum likelihood phylogenetic analysis of the nef sequence of 08MB26ZA. The sequence clusters with HIV-1 subtype C reference sequences. Bootstrap values above $70 \%$ are shown. The tree is rooted with group O sequences. The scale represents the number of base substitutions per site 


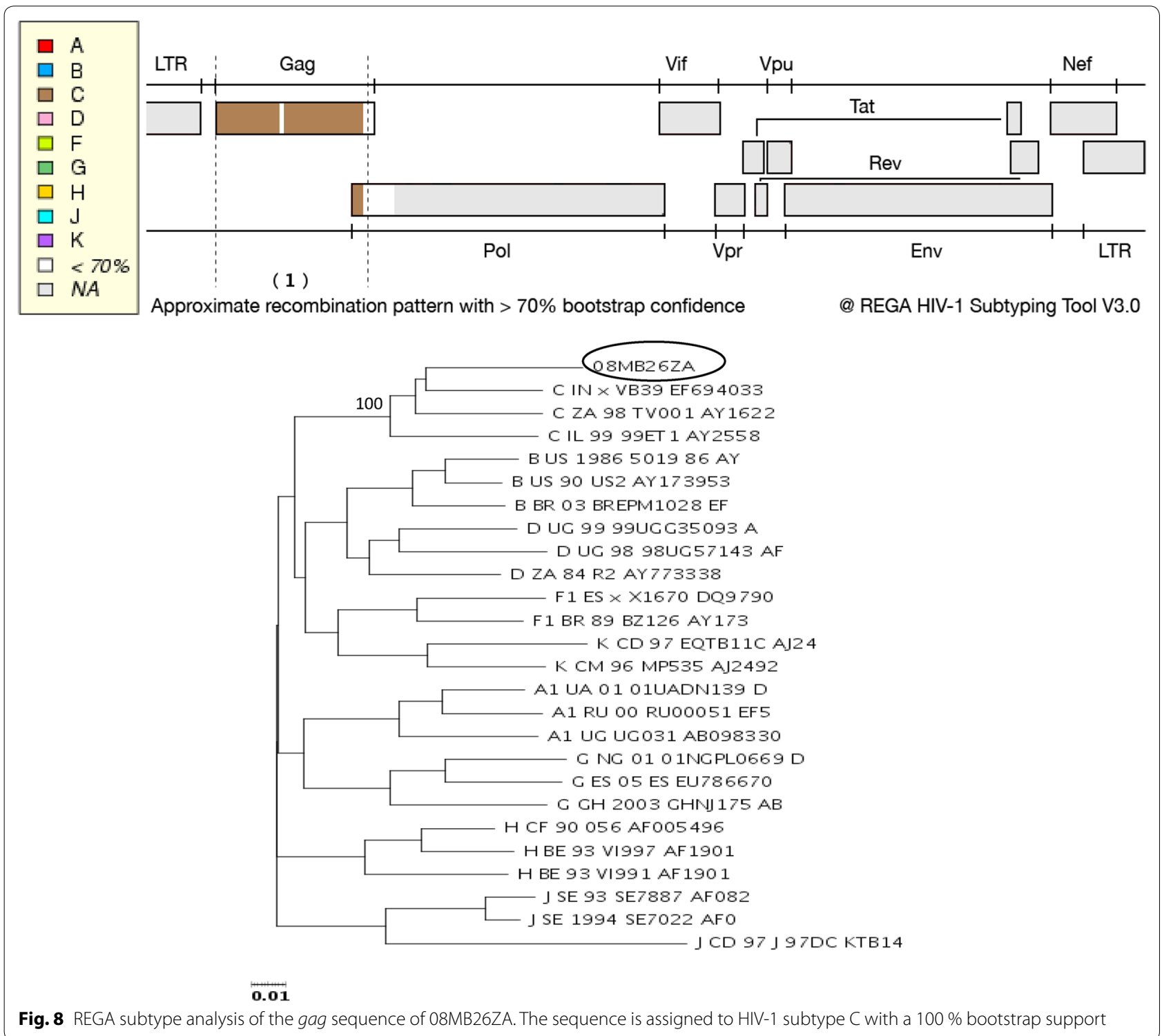

(See figure on next page.)

Fig. 9 a REGA HIV subtype recombination bootscan pattern of the pol sequence of 08MB26ZA. The bootscan analysis shows the following patterns: Segment (1) nucleotide position 2252-3192 is assigned to subtype J with $98 \%$ bootstrap confidence; segment (2) nucleotide position 3192-3512 is assigned to subtype G with 91 \% bootstrap confidence; section (3) nucleotide position 3512-3952 is assigned to subtype J with 86 \% bootstrap confidence; and section (4) nucleotide position 4312-4792 is assigned to subtype C with $92 \%$ bootstrap confidence. b REGA HIV-1 subtype/CRF recombination analysis of the pol sequence of 08MB26ZA. Segment (1) nucleotide position 2252-3952 is assigned to HIV-1 CRF 11_CPX with a bootstrap support of $100 \%$; segment (2) nucleotide position 4312-4792 is assigned to HIV-1 subtype C with a bootstrap support of $89 \%$

search of the Los Alamos HIV sequence database did not reveal any variant that contains HIV-1 subtype C and CRF11_cpx sequences in its genomic composition. In another vein, since population based sequencing was employed it is not possible to rule out dual infection with HIV-1 subtype C and CRF11_cpx. However, CRF11_cpx has not been reported from South Africa. It is also understood that the patient from whom the virus under investigation was recovered had never travelled out of South Africa, increasing the likelihood that the observation could be an infection with a recombinant CRF11_cpx/ HIV-1-C like virus. It is important to note that this is a 
a
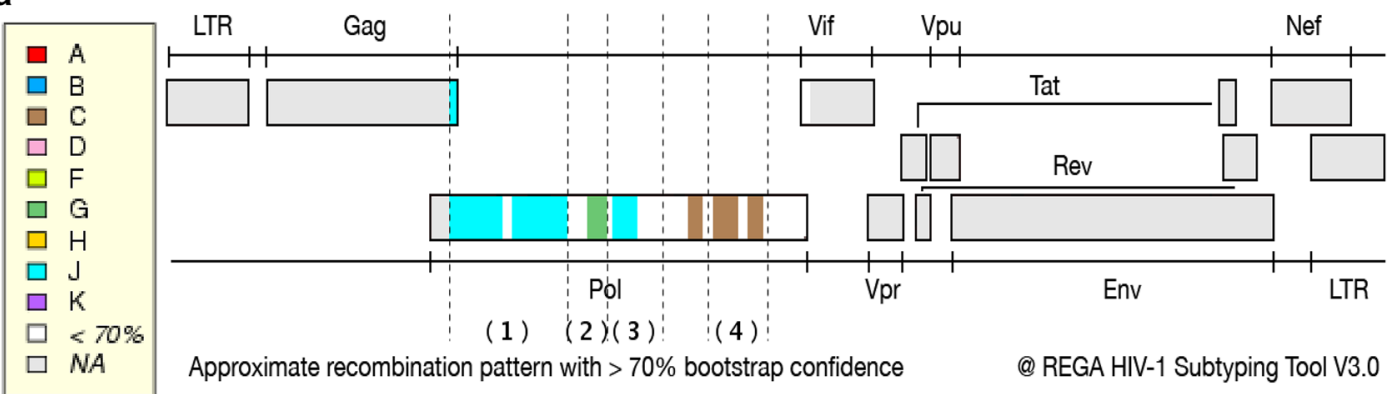

Approximate recombination pattern with $>70 \%$ bootstrap confidence

@ REGA HIV-1 Subtyping Tool V3.0

\begin{tabular}{|l|l|l|l|}
\hline Segment & Nucleotide position & Subtype assignment & Bootstrap value \\
\hline Segment 1 & $2252-3192$ & J & $98 \%$ \\
\hline Segment 2 & $3192-3512$ & G & $91 \%$ \\
\hline Segment 3 & $3512-3952$ & J & $86 \%$ \\
\hline Segment 4 & $4312-4792$ & C & $92 \%$ \\
\hline
\end{tabular}
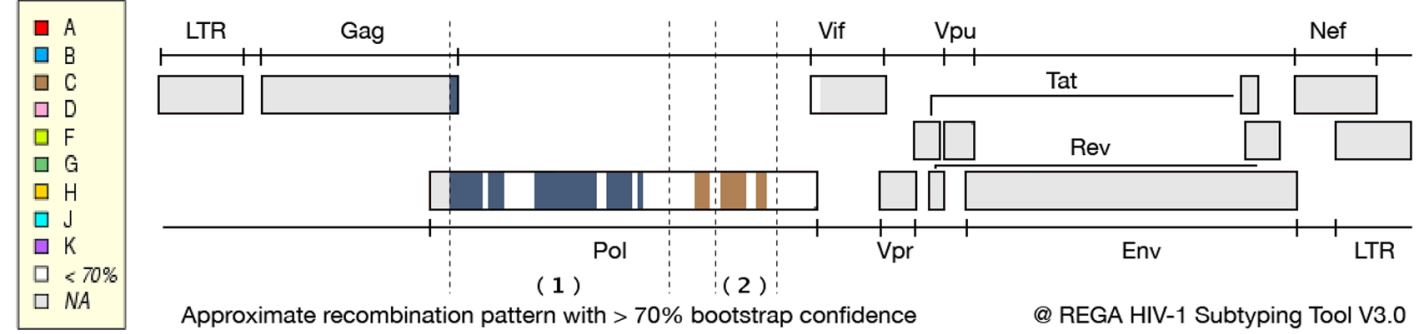

b

Segment (1): CRF11_cpx assignment

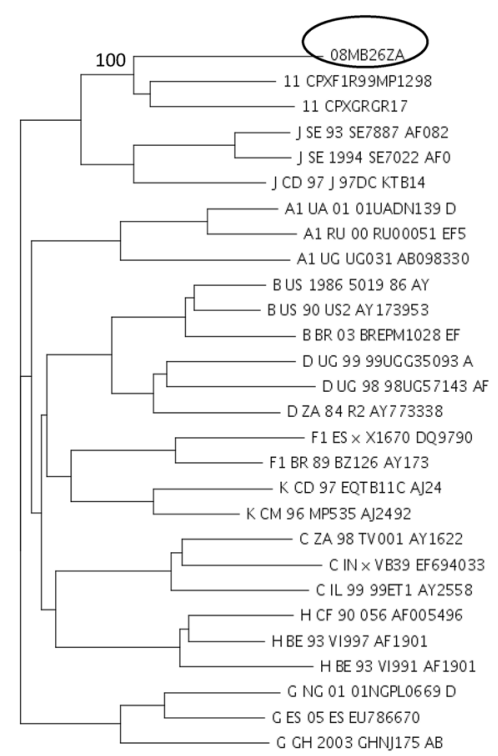

Segment (2): HIV-1 subtype C assignment

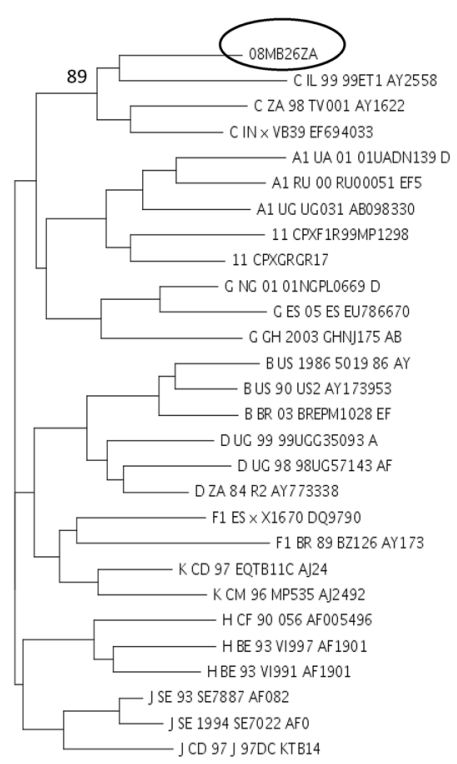

$\overline{0.01}$ 


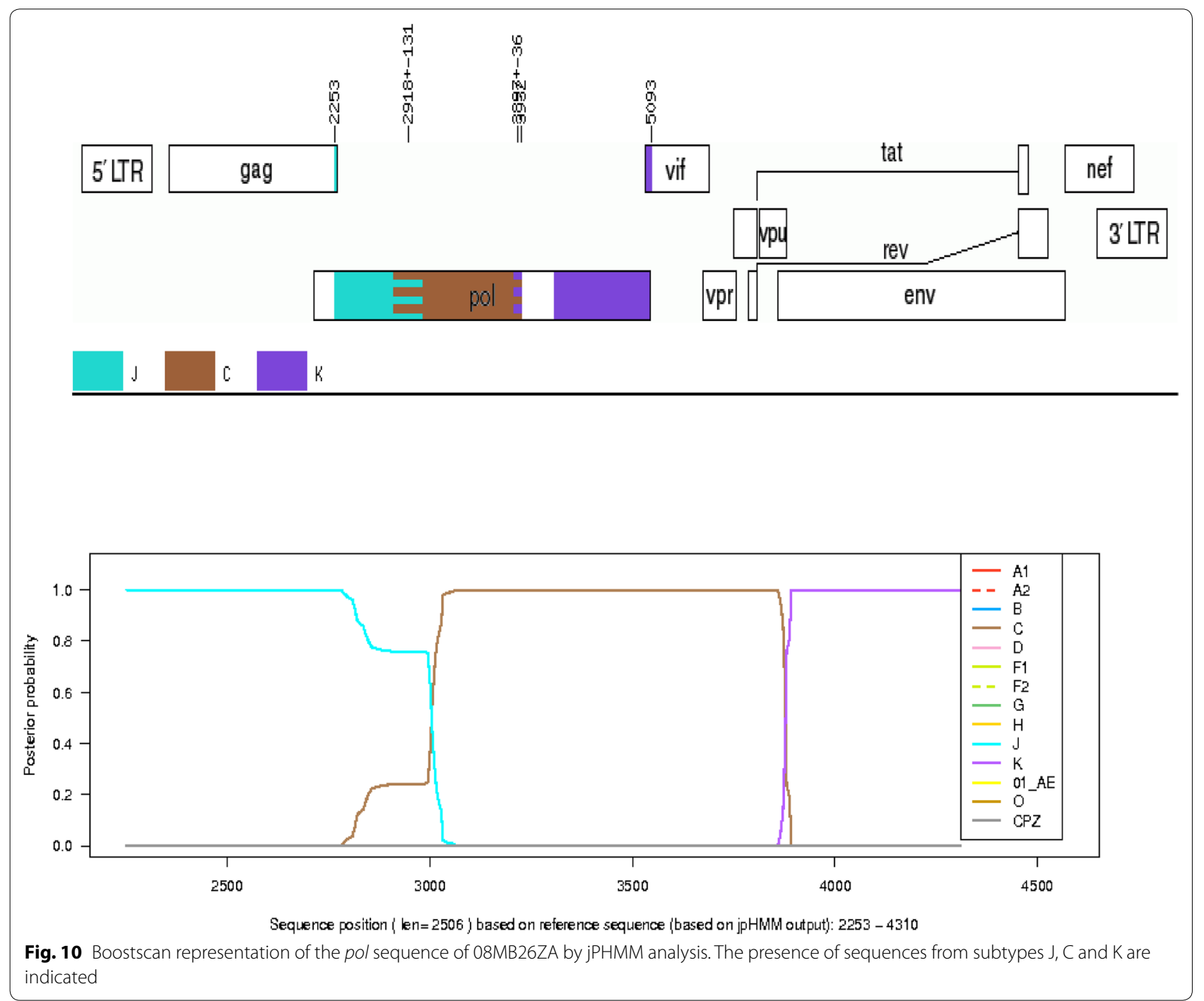

description of a virus from a single individual harboring genes from an HIV circulating recombinant form (CRF11_cpx) and a pure HIV subtype (HIV-1 subtype C). Therefore, the implications in terms of its epidemiologic and biologic preferences are unknown.

\section{Genotypic drug resistance and co-receptor usage analyses} Drug resistance genotypic analysis of the PR, RT and IN genes did not reveal the presence of primary or major drug resistance mutations. However, examination of the PR revealed the following amino acid substitutions; L10V, T12P, K14R, I15 V, G16A, A28P and T31R and V32L; while in the RT the following amino acid amino acid substitutions I5 V, E6D, V35T, T39L, K122 N, K173T, D177G, T200E, I202V, R211K, V245N, E248D, D250E, A272P, E293 V, I329 V, Q334L, G335D, R356K, M357R, R358K, G359A, K366R, T369A, A371V,
T377L, K390R, K395R, A400T, E435A, and A437V were observed in comparison with the global subtype B consensus sequence. Similarly, the following amino acid substitutions; K14R, S24N, D25E, V31I, M50I, I72V, V99F, F100Y, T112V, T124N, T125A, K136Q, V201I, Y227F, L234I, N254K, S255G, and S283G were observed in the IN when compared with the global subtype B consensus sequence. These amino acid changes are of unknown phenotypic significance. However, in the absence of primary or major drug resistant associated mutations it is expected that virus 08MB26ZA would be susceptible to currently used antiretroviral (ARV) drugs in South Africa.

The V3 loop is a major determinant for viral tropism and co-receptor usage. Analysis of the V3 loop of 08MB26ZA showed it had the GPGQ tetrapeptide motif and co-receptor usage prediction with webPSSM showed 


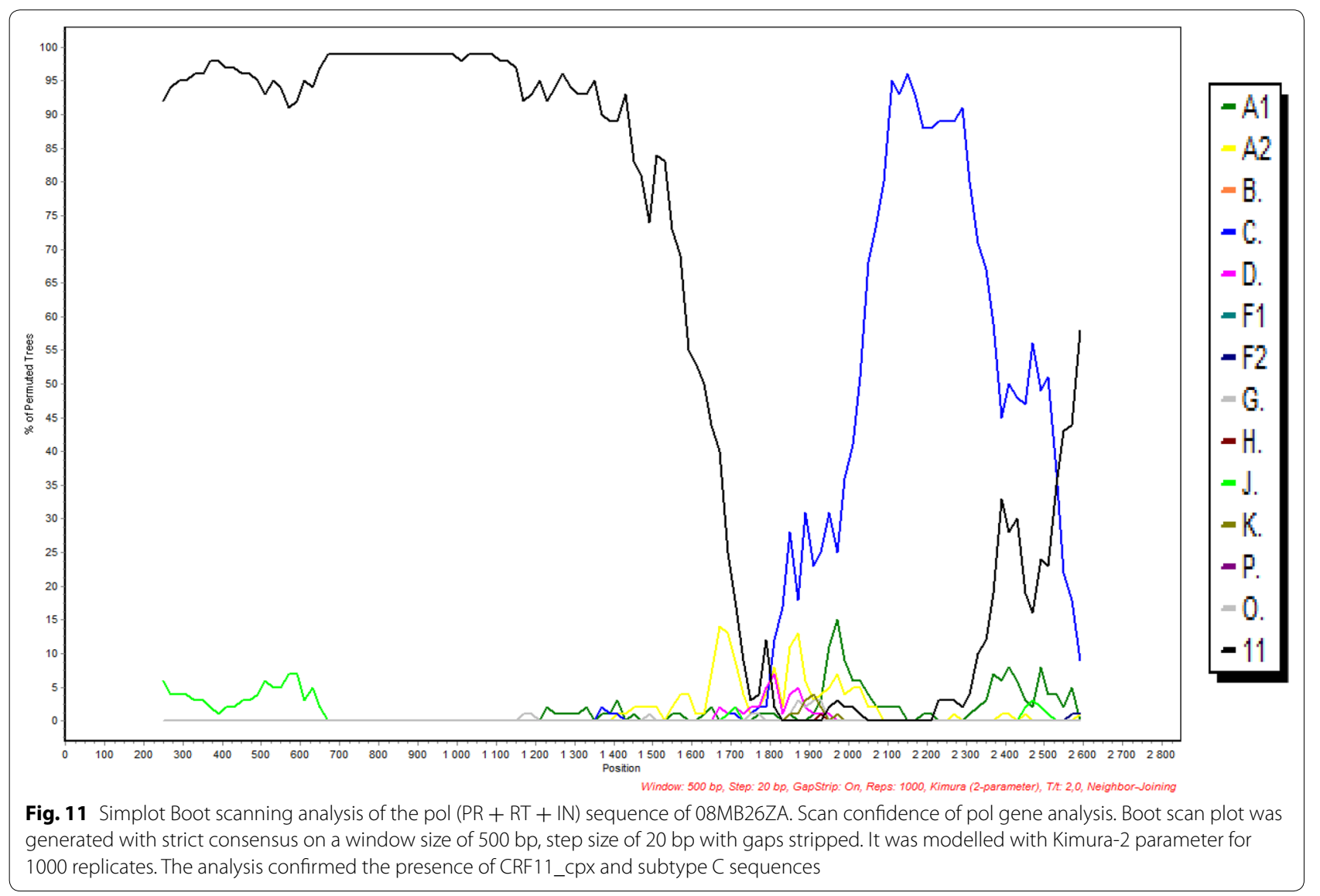

Table 1 Summary of subtype assignments by phylogenetic, REGA and jPHMM HIV subtyping and recombination analyses of 08MB26ZA

\begin{tabular}{llll}
\hline Gene & Phylogenetic analysis & REGA & jPHMM \\
\hline gag & HIV-1 subtype C & HIV subtype C & HIV-1 subtype C \\
PR & HIV-1 CRF1__cpx & HIV subtype J & HIV-1 subtype J \\
RT & HIV-1 CRF1__cpx & HIV-1 CRF11_cpx & HIV-1 subtype J/C \\
IN & Outlier to HIV-1 subtype C & HIV-1 subtype C & HIV-1 subtype C/A1 \\
pol & HIV CRF11_cpx & CRF11_cpx/HIV-1 subtype C & HIV-1 subtype J/C/K \\
nef & HIV-1 subtype C & HIV-1 subtype C & HIV-1 subtype C \\
env (C2-V5) & Outlier to HIV-1 subtype C & HIV-1 subtype C & HIV-1 subtype C \\
\hline
\end{tabular}

it was a CCR5 virus with a net charge of +3 . This coreceptor prediction suggests that the virus would be susceptible to entry inhibitors such as Maraviroc.

\section{Conclusion}

The identification of a CRF11_cpx/HIV-1 subtype C-like recombinant virus from South Africa, a region with an overwhelming predominance of HIV-1 subtype C, has been described. This is a signal suggesting the likely introduction of new and complex genetic variants in
South Africa; and highlights the importance for regular genetic diversity studies due to potential implications for diagnosis. Apparently, this is the first report of a putative CRF11_cpx/HIV-1 subtype $\mathrm{C}$ unique recombinant from South Africa.

\section{Methods}

\section{Patient information}

Blood was collected from a 47 year old female (08MB26ZA) from Capricorn district, South Africa in 
2008. At the time of blood collection, the patient was not on ARV. The CD4+ cell count and plasma viral load measurements were not available. The route of infection was heterosexual and the probable year of infection was 2005.

\section{Research ethics considerations}

The study protocol was approved by the Safety, Health and Research Ethics Committee of the University of Venda, South Africa. Signed informed consent was obtained before blood sample and demographic data were collected.

\section{Generation of gene regions by PCR}

Viral DNA was obtained from patient's peripheral blood mononuclear cells using QIAamp DNA kit (Qiagen, Valencia, CA) according to the manufacturer's instructions. The gag, pol, env $\mathrm{C} 2-\mathrm{V} 5$ and nef genes were amplified by nested PCR. The first round PCR of the different fragments were amplified using one of the following primer pairs; GagDrev 5'-AAT TCC TCC TAT CAT TTT TGG $-3^{\prime}$ and Gag Dforw 5'-TCT CTA GCA GTG GCG CCC G-3' for gag; Pol1C; $5^{\prime}$-GAA GGA CAC CAA TTG AAA GAC TGC AC-3' and INrev1; $5^{\prime}$-TCT CCT GTA TGC AGA CCC CAA TAT-3' for pol, while ED5;5'-ATG GGA TCA AAG CCA TGT G-3' and ED12; 5'-ATG GCT TCC TGC TCC CAA GAA CCC AAG-3' were used for the env $\mathrm{C} 2$-V5; and SQ15FC; $5^{\prime}$-GAG AGC GGT GGA ACT TCT- $3^{\prime}$ and NefOR; $5^{\prime}$-AGG CAA GCT TTA TTG AGG-3' for the nef gene.

The amplifications were carried out in a reaction mixture containing $5 \mu \mathrm{l}$ of $10 \times$ buffer, $200 \mu \mathrm{M}$ of dNTPs, $0.5 \mu \mathrm{M}$ of each primer, $0.5 \mathrm{U}$ of Taq Expand Long Template, and $10 \mu \mathrm{l}$ of DNA template in a final reaction volume of $50 \mu \mathrm{l}$. The cycling conditions used for all the reactions were as follows: $94{ }^{\circ} \mathrm{C}$ for $3 \mathrm{~min}$ followed by 35 cycles of $94{ }^{\circ} \mathrm{C}$ for $30 \mathrm{~s}, 55^{\circ} \mathrm{C}$ for $45 \mathrm{~s}$ and $72{ }^{\circ} \mathrm{C}$ for $2 \mathrm{~min}$; and a final extension for $10 \mathrm{~min}$ at $72{ }^{\circ} \mathrm{C}$. The nested reaction was carried out in a reaction mixture and cycling conditions as outlined above but with the following primer pairs Gag Aforw 5'-CTC TCG ACG CAG GAC TCG GCT T-3' and GagCrev; 5'-TCTTCTAATAC TGTATCATCTGC-3' for the gag gene; GagP1 5'-C AAGGG GAGGCCAGGGAATTT- $3^{\prime}$ and INrevII $5^{\prime}$-C CTAGTGGGATGTGTACTTCTGA- $3^{\prime}$ for the pol gene; ES7 5'-CTGTTAAATGGCAGTCTAGC- $3^{\prime}$ and ES8 5'-CACTTCTCC AATTGTCCCTCA-3' for env C2-V5; and Nefforw 5'-CCTAGAAGAATAAGACAG GGCT T- $3^{\prime}$ and Nefrev $5^{\prime}$-CCTGGAACGCCCCAGTGG-3' for the nef gene region. The amplified products were purified and directly sequenced using a BigDye Terminator sequencing kit (Applied Biosystems, Foster City, CA) making use of appropriate sequencing primers for the $g a g$ and pol genes while the $e n v$ and nef were sequenced with their nested primers. The sequenced fragments from both strands were assembled and edited using SeqMan Pro and Seqbuilder program contained in the DNAStar software version 7 (DNASTAR, INC, Madison, Wisconsin, USA).

\section{Phylogenetic and recombination analyses}

The gag, pol, PR, RT, IN, env C2-V5, and nef sequences were aligned with pure subtypes $\mathrm{A}-\mathrm{D}, \mathrm{F}-\mathrm{H}, \mathrm{J}, \mathrm{K}$, and CRF11_cpx reference sequences obtained from the Los Alamos database. Test and reference sequences were codon aligned using Muscle and phylogenetic trees generated by the maximum likelihood method as implemented in MEGA version 5.2 with 1000 bootstrap replicates to estimate the reliability of the branching clusters (Tamura et al. 2011). The gag and pol sequences were additionally analyzed for recombination and CRF with REGA HIV Subtyping tool version 3.0 (Peña et al. 2012). The REGA tool employs phylogenetic and bootscanning methods to assess the query sequence against a mirror of pure subtypes and CRFs. Additionally, the pol sequence was analysed by jPHMM and Simplot to confirm recombination.

\section{Determination of genotypic drug resistance and co-receptor usage}

The PR, RT and IN nucleotide sequences were also submitted to the Stanford HIV Drug Resistance Interpretation Algorithm for the detection of mutations associated with drug resistance and closest subtype identity (Stanford HIV drug resistance mutation database). The V3 loop of the env gene is a major determinant for viral tropism and co-receptor usage. The predicted amino acid sequences of the V3 loop of virus 08MB26ZA was submitted to the webPSSM, an online interactive program that predicts co-rector usage of viruses by calculating the net charge of the V3 loop and makes predictions on probable co-receptor usage (WebPSSM).

\section{GenBank accession numbers}

The gene regions described here have been deposited in GenBank under the following accession numbers: HM049909 (gag); GU188817 (PR); HM049908 (RT); HM049906 (IN); HM049910 (env C2-V5); HM049907 (nef).

\section{Authors' contributions}

Bl generated the nucleotide sequences. POB designed the study, analyzed the data and wrote the manuscript. Both authors read and approved the final manuscript.

\section{Author details}

1 HIV/AIDS and Global Health Research Programme, Room FF172 Life Sciences Building, Department of Microbiology, University of Venda, 
Thohoyandou 0950, South Africa. ${ }^{2}$ Department of Biochemistry and Microbiology, University of Fort Hare, Alice, South Africa.

\section{Acknowledgements}

The cooperation of the study participant is appreciated. This study was carried out with the support from a grant awarded to POB by the National Department of Health, under the Comprehensive HIV and AIDS Care, Treatment and Management Plan for South Africa. Additional support was obtained from the National Research Foundation, South Africa (Grant UID 80576 to POB). The opinions expressed here are those of the authors.

\section{Competing interests}

$\mathrm{BI}$ declares no conflict of interest. $\mathrm{POB}$ is an editorial advisory board member of SpringerPlus.

Received: 12 September 2015 Accepted: 22 February 2016

Published online: 05 March 2016

\section{References}

Bartolo LM, Epalanga M, Bartolomeu J, Fonseca M, Mendes A, Gama A, Taveira $N$ (2009) High genetic diversity of human immunodeficiency virus type 1 in Angola. AIDS Res Hum Retroviruses 21:306-310

Bredell H, Hunt G, Casteling A, Cilliers T, Rademayer C (2002) HIV-1 subtypes A, $D, G, A G$ and unclassified sequences identified in South Africa. AIDS Res Hum Retroviruses 18:681-683

Caron M, Kekana-Douki SE, Makuwa M et al (2012) Prevalence, genetic diversity and antiretroviral drug resistance-associated mutations among untreated HIV-1-infected pregnant women in Gabon, Central Africa. BMC Infect Dis 12:64. doi:10.1186/1471-2334-12-64

Corbet S, Muller-Trutwin MC, Versmisse P, Delarue S, Ayouba A, Lewis J, Brunak $S$ (2000) Env sequences of simian immunodeficiency viruses from chimpanzees in Cameroon are strongly related to those of human immunodeficiency virus group N from the same geographic area. J Virol 74:529-534

Hemelaar J (2013) Implications of HIV diversity for the HIV-1 pandemic. J Infect 66:391-400

Hemelaar J, Gouws E, Ghys PD, Osmanov S (2011) WHO-UNAIDS network for HIV isolation and characterisation. Global trends in molecular epidemiology of HIV-1 during 2000-2007. AIDS 25:679-689

Iweriebor BC, Bessong PO, Mavhandu LG, Masebe TM, Nwobegahay J, Moyo SR, Mphahlele JM (2011) Genetic analysis of the near full-length genome of an HIV type $1 \mathrm{~A} 1 / \mathrm{C}$ unique recombinant form from Northern South Africa. AIDS Res Hum Retroviruses 27:911-915

Kiwanuka N, Layendecker O, Quinn TC, Wawer MJ, Shepherd J, Robb M, Kigozi G, Kagaayi J, Serwadda D, Makumbi FE, Reynolds SJ, Gray RH (2009) HIV-1 subtypes and differences in heterosexual HIV transmission among discordant couples in Rakai, Uganda. AIDS 23:2479-2484

Koyalta D, Charpentier C, Beassamda J, Rey E, Si-Mohamed A, Djemadji-Oudjeil N, Bélec L (2009) High frequency of antiretroviral drug resistance among HIV-infected adults receiving first-line highly active antiretroviral therapy in N'Djamena, Chad. Clin Infect Dis 49:155-159

Laukkanen T, Albert J, Littsola K, Green SD, Carr JK, Leitner T, McCutchan FE, Salminen MO (1999) Virtually full-length sequences of HIV type 1 subtype J reference strains. AIDS Res Hum Retroviruses 15:293-297

Liitsola K, Tashkinova I, Laukkanen T, Korovina G, Smolskaja T, Momot O, Mashkilleyson N (1998) HIV-1 genetic subtype A/B recombinant strain causing an explosive epidemic in injecting drug users in Kaliningrad. AIDS 12:1907-1919

Los Alamos National Laboratory database (2014) http://www.hiv.lanl.gov/ content/index. Accessed 15 Dec 2013

Montavon C, Vergne L, Bourgeois A, Mpoudi-Ngole E, Malonga-Mouellet G, Butel C, Toure-Kane C, Delaporte E, Peeters M (2002) Identification of a new circulating recombinant form of HIV type 1, CRF11-cpx, involving subtypes A, G, J, and CRF01-AE, in Central Africa. AIDS Res Hum Retroviruses 18:231-236
Ndembi N, Abraha A, Pilch H, Ichimura H, Mbanya D, Kaptue L, Salata R, Arts EJ (2008) Molecular characterization of human immunodeficiency virus type 1 (HIV-1) and HIV-2 in Yaoundé, Cameroon: evidence of major drug resistance mutations in newly diagnosed patients infected with subtypes other than subtype B. J Clin Microbiol 46:177-184

Nwobegahay JM, Bessong PO, Masebe TM, Mavhandu LG, Iweriebor BC, Selabe G (2011) Prevalence of antiretroviral drug resistance mutations and HIV-I subtypes among newly-diagnosed drug-naïve persons visiting a voluntary testing and counselling centre in northeastern South Africa. J Health Popul Nutr 29:303-309

Paraskevis D, Magiorkinis M, Paparizos V, Pavlakis GN, Hatzakis A (2000) Molecular characterization of a recombinant HIV type 1 isolate (A/G/E/?): unidentified regions may be derived from parental subtype $E$ sequences. AIDS Res Hum Retroviruses 16:845-855

Peña ACP, Faria NR, Imbrechts S, Libin P, Abecasis AB, Deforche K, Gomez A, Camacho RJ, de Oliveira T, Vandamme AM (2012) Performance of the subtyping tools in the surveillance of HIV-1 epidemic: comparison between Rega Version 3 and six other automated tools to identify pure subtypes and circulating recombinant forms. In: Proceedings of the 17th international bioinformatics workshop on virus evolution and molecular epidemiology. University of Belgrade, Faculty of Medicine, Belgrade, Serbia, 27-31 Aug 2012

Ramirez BC, Simon-Loriere E, Galetto R, Negroni M (2008) Implications of recombination for HIV diversity. Virus Res 134:64-73

Tamura K, Peterson D, Perterson N, Stecher G, Nei M, Kumar S (2011) MEGA5: molecular evolutionary genetics analysis using maximum likelihood, evolutionary distance, and maximum parsimony methods. Mol Biol Evol 28:2731-2739

Taylor BS, Sobieszczyk ME, McCutchan FE, Hammer SM (2008) The challenges of HIV-1 subtype diversity. N Engl J Med 358:1590-1602

Torimiro JN, D'Arrigo R, Takou D, Nanfack A, Pizzi D, Ngong I, Carr JK, Joseph FP, Perno CF, Cappelli G (2009) Human immunodeficiency virus type 1 intersubtype recombinants predominate in the AIDS epidemic in Cameroon. New Microbiol 32:325-331

Trask SA, Derdeyn CA, Fideli U, Chen Y, Meleth S, Kasolo F, Musonda R, Hunter E, Gao F, Allen S, Hahn BH (2002) Molecular epidemiology of human immunodeficiency virus type 1 transmission in a heterosexual cohort of discordant couples in Zambia. J Virol 76:397-405

WebPSSM. http://indra.mullins.microbiol.washington.edu/pssm/webpssm3.pl. Accessed 30 Nov 2013

Wilbe K, Casper C, Albert J, Leitner T (2000) Identification of two CRF11-cpx genomes and two preliminary representatives of a new circulating recombinant form (CRF13_cpx) of HIV type 1 in Cameroon. AIDS Res Hum Retroviruses 18:849-856

Wilkinson E, Engelbrecht S (2009) Molecular characterization of non-subtype $\mathrm{C}$ and recombinant HIV-1 viruses from Cape Town, South Africa. Infect Genet Evol 9:840-846

Yebra G, de Mulder M, Holguín A (2013) Description of HIV-1 group M molecular epidemiology and drug resistance prevalence in Equatorial Guinea from migrants in Spain. PLoS One. doi:10.1371/journal.pone.0064293

\section{Submit your manuscript to a SpringerOpen ${ }^{\circ}$ journal and benefit from:}

- Convenient online submission

- Rigorous peer review

- Immediate publication on acceptance

- Open access: articles freely available online

- High visibility within the field

- Retaining the copyright to your article

Submit your next manuscript at springeropen.com 